\title{
Stabilization of combustion front in supersonic flow using streamer's discharge
}

\author{
Pavel Bulat ${ }^{1,2}$, Lev Grachev ${ }^{3}$, Igor Esakov ${ }^{3}$, Vladimir Upyrev ${ }^{1,2^{*}}$ \\ ${ }^{1}$ Baltic State Technical University "Voenmeh", 190005 Saint-Petersburg, Russia \\ ${ }^{2}$ Saint Petersburg National Research University of Information Technologies, Mechanics and Optics, \\ 197101 Saint-Petersburg, Russia \\ ${ }^{3}$ Moscow Radiotechnical Institute of the Russian Academy of Sciences, 117519 Moscow, Russia
}

\begin{abstract}
Thermodynamic analysis shows that for flights with velocities exceeding six sound velocities, it is required to burn fuel not in a subsonic but in a supersonic flow. The aim of this work is to investigate the possibility of creating a stationary combustion front in a supersonic flow by igniting the mixture with an attached microwave discharge. Discharges are created on the resonator by means of a pulsed source of quasi-optical microwave radiation. This method of initiation is one or two orders of magnitude more economical than other known methods of plasma ignition and combustion stabilization. A numerical evaluation and comparison with experiment of the propagation velocity of a subcritical streamer discharge in a stationary medium and in a supersonic drifting flow are performed. Experiments have been conducted to ignite a flat flow of propane-air mixture, as well as ignition of the propane stream fed into the airflow, which simulates the operation of the fuel injector. In all cases, the experiments confirmed a steady fuel combustion, which was controlled by the temperature measurements with a thermocouple.
\end{abstract}

\section{Introduction}

The issue of initiation and maintenance of combustion in ramjet air-jet engines, including engines with supersonic combustion, is considered. At the moment, such problems are solved by placing in the flow of poorly streamlined bodies and the stabilization of combustion in the circulation zones by such bodies. This leads to an additional resistance, which increases as the flight speed and the speed of the ignited fuel mixture increase. An alternative variant of ignition of a mixture by means of a plasma discharge, a streamer and an attached diffuse one, is considered in this work. Due to the use of the resonant method of amplifying the electric field, these discharges ignite at the initial field strength much lower than the breakdown energy $E_{c r}$. Detailed studies of such discharges carried out during the last 20 years at the RAS of the Russian Academy of Sciences have shown that the abovementioned method is 20 to 80 times higher in terms of energy costs than other methods, including corona discharge and spark ignition $[1,2]$.

\footnotetext{
* Corresponding author: upyrevvv@yandex.ru
} 
The paper studies the initiation of combustion of a plane premixed air and propane stream at different speeds and fuel-oxidant ratios, as well as ignition of the propane stream fed into the air flow, similar to the operation of the fuel injector. Also in the paper the semiempirical and numerical modeling of subcritical streamer discharge and deeply subcritical attached diffuse discharge is considered.

\section{Mathematical model}

When the microwave field is turned on and when free electrons are present in this region, their number increases with time $t$, and the region occupied by the discharge plasma diffusely "blur". This process at the initial stage is described by the following equation for the electron density

$$
\frac{\partial n_{e}}{\partial t}=D_{e} \cdot \frac{\partial^{2} n_{e}}{\partial r^{2}}+\left(v_{i}-v_{a}\right) \cdot n_{e}
$$

where $n_{e}$ - concentration of electrons, $D_{e}=7500 / p$ - electron diffusion coefficient, $p-$ gas pressure in $\mathrm{Pa}, t-$ time, $v_{i}$ - ionisation frequency, $v_{a}$ - frequency of attachment of electrons to atoms, $r$ - radius vector. In the focus of the microwave beam, in contrast to the constant field, the average electron velocity in the avalanche is zero. Proceeding from this, it is possible to obtain the propagation velocity of the plasmoid boundary [3]:

$$
u_{s}=2 \sqrt{D_{e} \cdot\left(v_{i}-v_{a}\right)}+\frac{|\mu| \cdot E}{\omega+\left(v_{i}-v_{a}\right)}\left(v_{i}-v_{a}\right)
$$

where the first term is determined by electron diffusion, and the second by their drift in an electric field along the tension vector $E, \mu$ - mobility of electrons, $\omega$ - circular microwave radiation frequency.

In a motionless gas the problem is simplified and it is possible to estimate the velocity of the streamer propagation, based on the following considerations. Since outside the diffuse discharge $E<E_{c r}$, then for the development of the streamer the density of the gas inside it must fall, otherwise deionization will occur and the channel will lose the ability to increase the field strength to $E>E_{c r}$. Studies [4] show that the level of supercriticality $E$ / $E_{c r}$, realized at the streamer head, is set at a level slightly higher than one, at which the velocity of the ionization front corresponds to the rate of channel heating directly at the head of the streamer. Thus, the second term in Eq. (2) is negligibly small and it can be ignored when estimating the growth rate of a subcritical streamer. The expression for the head speed of the streamer

$$
u_{s}=2 \sqrt{D_{e} \cdot \frac{E_{c r}^{2}}{c_{p} \cdot p} \cdot \frac{\omega}{4 \cdot \pi \cdot \ln \left(\frac{E_{c r}}{E}\right)}},
$$

where $c_{p}-$ specific heat at constant pressure.

\section{Streamer velocity}

Figure 1 shows the results of calculating the speed and radius of the streamer in comparison with the experimental data obtained when shooting discharges with a high-speed Phantom v.251 camera at a speed of 510000 frames / s. Figure 2 illustrates the influence of 
supersonic air flow on a subcritical microwave discharge. The streamer microwave discharge practically does not change its shape, but its plasma channels are blurred.



a)



b)

Fig. 1. The propagation velocity of the streamer discharge in a front free space (a) and in a quartz tube (b) at atmospheric pressure, depending on the field strength in the region of the initiator: (-) calculation by formula (4), ( $\square \circ)$ - the results of the experiment.
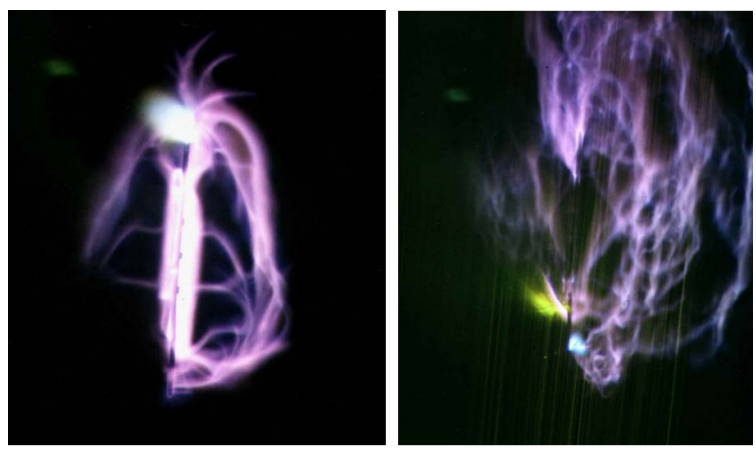

Fig. 2. Subcritical streamer discharge in still air (a) and in a supersonic flow at the velocity $V=500$ $\mathrm{m} / \mathrm{s}(\mathrm{b})$. Air pressure $p=13.3 \mathrm{kPa}$, field strength $E_{0}=1.7 \mathrm{kV} / \mathrm{cm}$.

\section{Experimental setup}

The scheme of the experimental setup is shown in Figure 3. Installation is a pulsed vacuumtype wind tunnel. The premixed propane mixture with air exits the nozzle at a speed of 10 to $500 \mathrm{~m} / \mathrm{s}$. The speed is controlled by the total pressure in the receiver. In the first case, a cylindrical vibrator with rounded ends was used to initiate combustion (Fig. 3a). In the second, a tubular initiator with a quartz head (Fig. 3b), through which propane was pumped. The tube itself was placed in the external air flow instead of the cylindrical initiator of the installation figure 3a. The thermocouple and the pitot tube were installed at the stagnation point. 


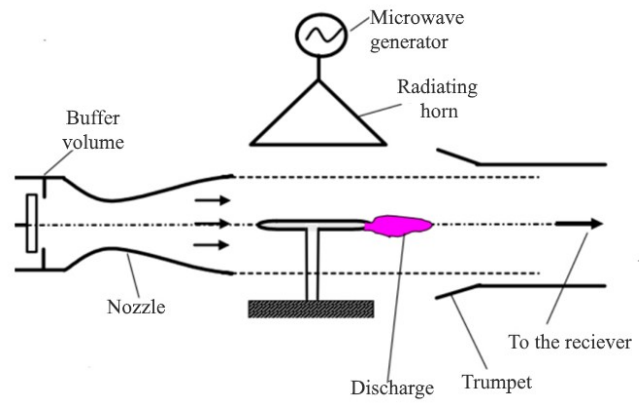

a)

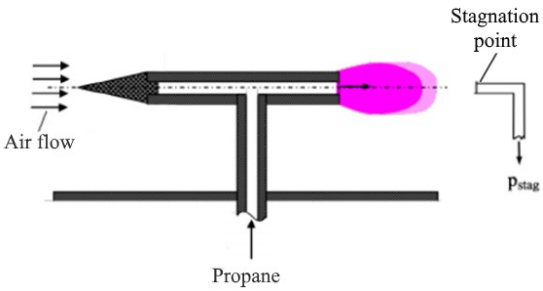

b)

Fig. 3. The scheme of the experimental setup.

\section{Results}

The experimental results for the premixed fuel mixture are shown in Fig. 4. Using the cap, the flow is flat. Its speed is $30 \mathrm{~m} / \mathrm{s}$. The temperature at the stagnation points is shown to the right. The flame is visible even for the case of a particularly poor mixture (Fig. 4e). In [4], we have already investigated the maintenance of supersonic combustion with the aid of an attached microwave discharge in a plane flow.
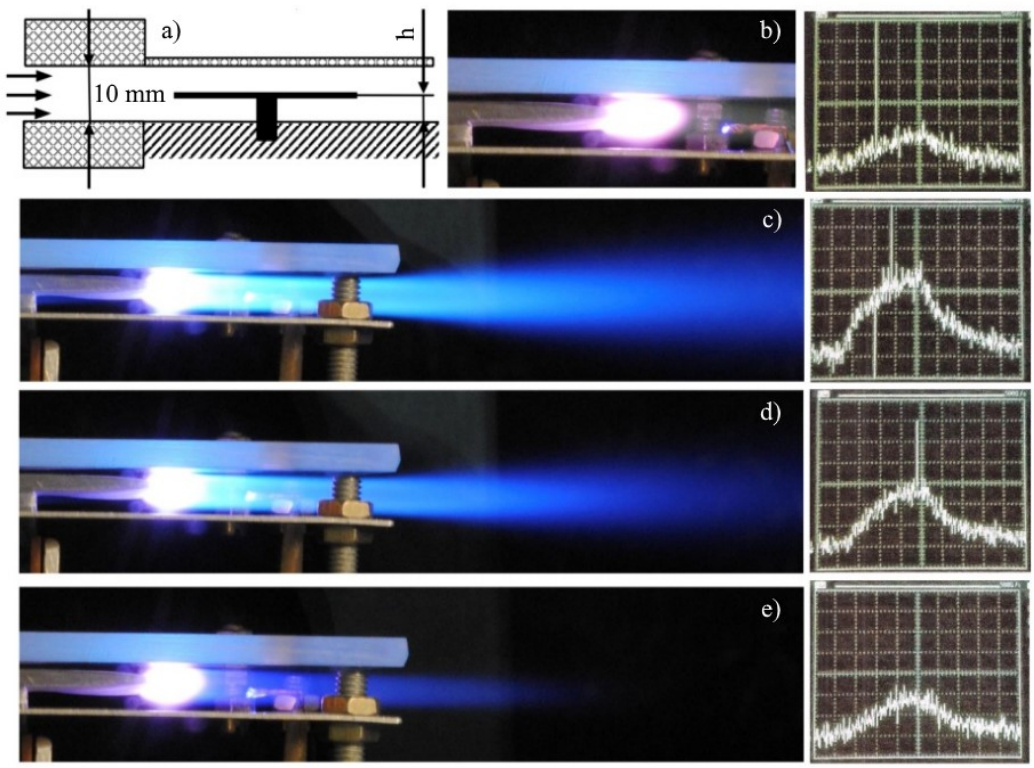

Fig. 4. The connected discharge in the mixture of propane with air at a flow rate of $30 \mathrm{~m} / \mathrm{s}$ : a) - the discharge initiator scheme, $b$ ) - discharge in the air, c) combustion of the stoichiometric mixture, fuel surplus factor $\alpha=1$, initial pressure $\mathrm{p}=3.3 \mathrm{bar}$; ) - combustion of the lean mixture, $\alpha=0.7, p=1$ bar; e) - combustion of a very poor mixture at $\alpha=0.5, \mathrm{p}=0.3 \mathrm{bar} . \mathrm{h}=5 \mathrm{~mm}$. Vertical division scale $100{ }^{\circ} \mathrm{C}$.

The results of the experiment for propane injection into the wake air flow through the tubular resonator are shown in Fig. 5. It is seen that, despite the weakening of the flame, combustion continues at supersonic speeds. 


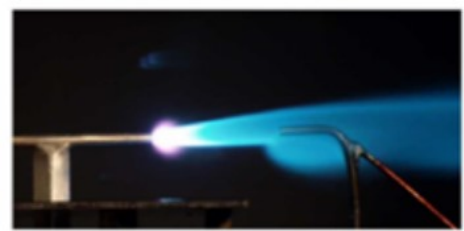

$12 \mathrm{~m} / \mathrm{s}$

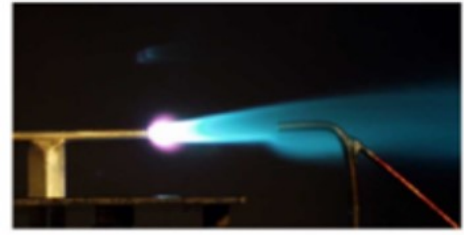

$30 \mathrm{~m} / \mathrm{s}$

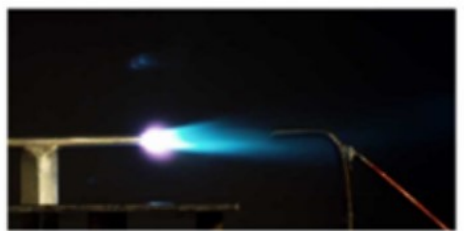

$85 \mathrm{~m} / \mathrm{s}$

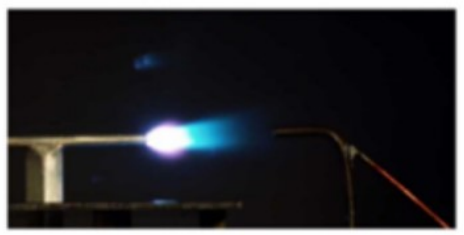

$200-500 \mathrm{~m} / \mathrm{s}$

Fig. 5. Combustion of propane in the cocurrent air flow by means of a subcritical microwave discharge at various airflow rates.

In Fig. 6 shows the temperature field in the cross section of the flow at a distance of 1.4 $\mathrm{cm}$ from the feed cut of the mixer. Data are taken for supersonic flow velocity. Noticeable temperature increase of 70-100 degrees Celsius.
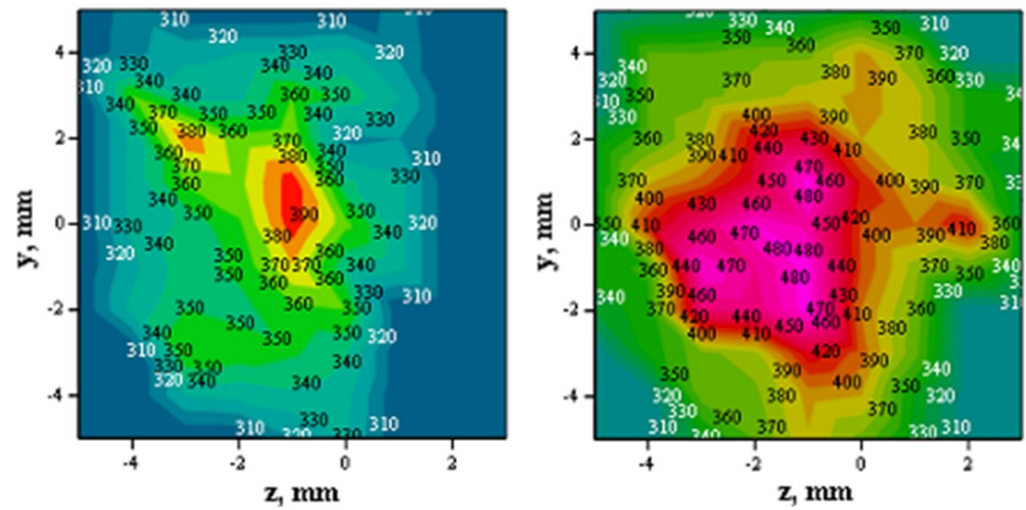

Fig. 6. Temperature in the cross section of the supersonic flow at a distance of $1.4 \mathrm{~cm}$ from the feed cut of the mixer; (a) - air is injected into the discharge area through the mixer, (b) - an air-propane mixture is injected into the discharge area.

\section{Conclusion}

Calculations have shown that the velocity of the streamers is several $\mathrm{km} / \mathrm{s}$, so they are not blown off by a supersonic flow. Experiments have been conducted to ignite the flow of a mixture of propane with air by various discharges at speeds up to twice the speed of sound. In all cases, the experiments confirmed a steady burning of the fuel, which was controlled from the temperature measurements with a thermocouple.

The work was supported by the Ministry of Education and Science of the Russian Federation (agreement No. 14.577.21.0277, unique identifier of the project RFMEFI57717X0277). 


\section{References}

1. P.V. Bulat, I.I. Esakov, L.P. Grachev, P.V. Denissenko, M.P. Bulat, I.A. Volobuev, Sc. and Tech. J. of ITMO 17, 569 (2017)

2. M.P. Bulat, P.V. Bulat, P.V. Denissenko, I.I. Esakov, L.P. Grachev, K.N. Volkov, I.A. Volobuev, Acta Astronaut. (2017)

3. A.M. Hovatson, An Introduction to Gas Discharges (Pergamon Press, 1976)

4. K.V.Khodataev, B.R.Gorelik, Plasma Phys. 23, 236 (1997)

5. V.V. Upyrev, I.I. Esakov, L.P. Grachev, Noneq. Proc. in phys. and chem., 1, 208 (2016) 